\title{
A Simple Physical Model to Assist in Fluid Mechanics Calculations
}

\section{Dr. Anne Dudek Ronan P.E., New York University}

Anne Dudek Ronan, Ph.D., P.E., is an Industry Professor in the Department of Civil and Urban Engineering NYU. Although her main area of interest is Water Resources Engineering, she teaches across the curriculum - from the freshman Introduction to Civil Engineering course to graduate classes in Groundwater Hydrology and Surface Water Pollution. She also advises PhD and Masters degree students and is faculty adviser for two student clubs. Previously, Anne was an Adjunct Professor at The Cooper Union and Assistant Professor at San Jose State University. She has won several teaching awards for her passion for undergraduate and graduate education. 


\section{A simple physical model to assist in fluid mechanics calculations}

This "5-minute presentation" introduces session participants to a simple physical model that the author uses to help students learn how to calculate the hydrostatic force on a planar surface. A frequent source of confusion among students when learning this concept is the definition of "area." Students often mix up the area of the planar surface with the area of the side of the pressure distribution. This model helps differentiate between the two. It also provides an easy means of showing students that the pressure distribution is not prismatic when the it acts on a planar surface of variable width. Since introducing this model several years ago, student performance on homework and exam problems related to this concept have generally increased by approximately ten percent.

Introduction

Physical models are widely viewed as a useful means of improving student learning. In civil engineering education, most physical models are full-size or scaled versions of a physical object. They are used to either help understand complicated three-dimensional geometry or demonstrate the response of the object to applied forces. The author has a built a simple model which does not represent a physical object; rather the model demonstrates the hydrostatic pressure distribution exerted by a liquid on a flat surface. The motivation for the model was the desire to help students avoid common errors when calculating the resultant force produced by the hydrostatic pressure distribution on a submerged flat surface.

\section{Underlying fluid mechanics concept}

Hydrostatic pressure in a liquid increases linearly with distance below the liquid surface. This is often represented by a triangular pressure distribution drawn on a vertical section through the flat plane on which the pressure is acting. The plane can be either vertical or inclined, so the section represents the plane by either a vertical or inclined line as shown in Figure 1.
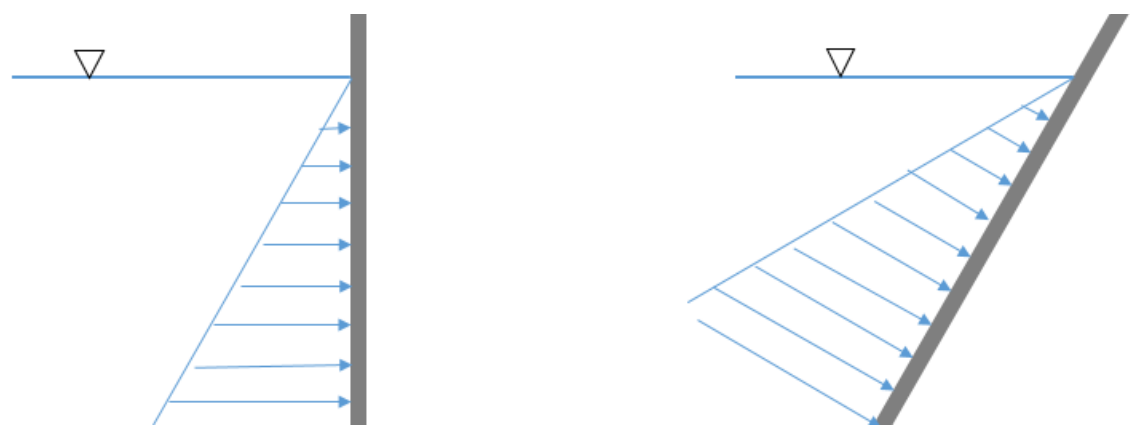

Figure 1: Pressure distributions on vertical and inclined planes

Most students in a fluid mechanics class have already taken a statics class, so they readily see that the triangular pressure distribution is a form of a distributed load. They often remember that they needed to integrate the distributed load to determine the resultant in statics class, so they 
assume that the area of the triangle is important for hydrostatic force problems. This is not always the case since pressure has dimensions of $\left[\mathrm{F} / \mathrm{L}^{2}\right]$ whereas distributed loads are often presented in dimensions of $[\mathrm{F} / \mathrm{L}]$.

If the planar area on which the pressure distribution is acting begins at the liquid surface and has a constant width (either a rectangle or other parallelogram oriented so two of the parallel sides are horizontal), the resultant force is simply equal to the area of the triangular pressure distribution multiplied by the constant width. If the planar area has constant width but its horizontal top edge is located below the liquid surface, the resultant force is the area of the part of the triangular pressure distribution which acts on the planar surface, which is trapezoidal as shown in Figure 2, multiplied by the constant width. The latter case is referred to as pressure acting on a submerged plane. In both cases, determining the resultant is equivalent to calculating the volume of a pressure prism.

Although not presented this way in textbooks, the resultant can be calculated using equation (1):

$$
R=A_{T} \cdot b
$$

where $R$ is the resultant force $[\mathrm{F}], A_{T}$ is the area of the triangle or trapezoid $[\mathrm{F} / \mathrm{L}]$, and $b$ is the uniform width of the planar area on which the pressure distribution is acting [L]. The dimensions of $A_{T}$ are $[\mathrm{F} / \mathrm{L}]$ because the sides of the triangle or trapezoid have dimensions of pressure and length.

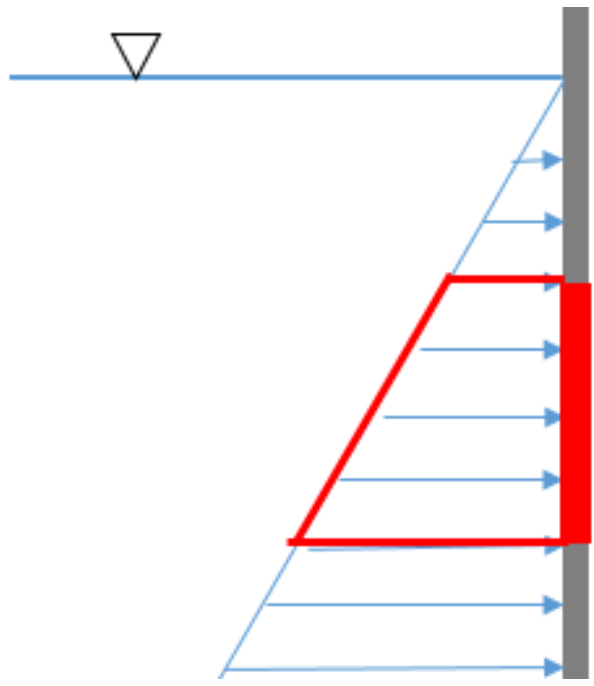

Figure 2. Pressure distribution acting on a submerged planar surface.

If the submerged planar area does not have a constant width, the triangular or trapezoidal area is not useful for calculating the resultant. In this case, equation (2) must be used:

$$
R=\gamma h_{C} A
$$

where $\gamma$ is the specific weight of the fluid $\left[\mathrm{F} / \mathrm{L}^{3}\right], h_{c}$ is the vertical distance from the liquid surface to the centroid of the planar area on which the pressure distribution is acting [L], and $A$ is 
the area of the planar surface on which the pressure distribution is acting $\left[\mathrm{L}^{2}\right]$. This equation is equivalent to the statement that the resultant is the product of the pressure exerted at the centroid of the surface area of interest $\left(p_{c}=\gamma h_{c}\right)$ and the area of interest, $A$.

Equation (2) is a general equation which holds for all cases - constant width or variable width, submerged or not submerged, vertical or inclined plane.

The author's experience is that students are often confused when calculating resultants because the illustrations in textbooks show the side of the pressure distribution, which is triangular, so they always try to use $A_{T}$ in their calculations, even when using equation (2) where the variable $A$ is not the same as variable $A_{T}$.

\section{The physical model}

The physical model was built in an effort to help students differentiate between $A$ and $A_{T}$. It was built when the author was a participant in the ASCE ExCEEd (Excellence in Civil Engineering Education) workshop during July 2014 and has been used all six semesters that the author has taught fluid mechanics since then. Figure 3 shows the model as constructed at the workshop, and Figure 4 shows the pieces that were brought back to campus afterwards (without the wooden structural support). Although the model was more stable with the structural support, the current format is much lighter, and smaller, and can be easily carried to class across campus. It also benefits from a few handwritten annotations that were added after several semesters of use.
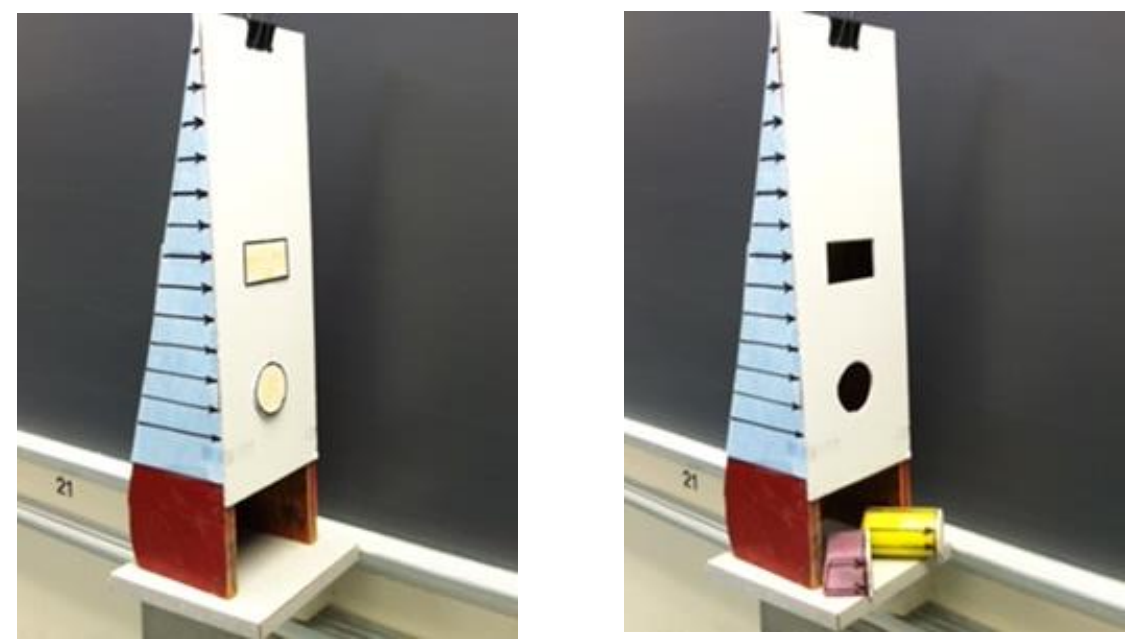

Figure 3. Physical model as constructed at ASCE ExCEEd workshop

The essential parts of the model were made from very simple materials: an 8" by 20" piece of white foam board, a triangular sheet of blue paper (from the cover of an exam blue book), two small pieces of foam, and tape. The triangular sheet of blue paper with arrows representing the hydrostatic pressure distribution below the liquid surface is taped to the foam board along one edge. The foam board has two shapes cut out of it below the line representing the liquid surface a horizontal rectangle and a circle. These shapes are the planar areas on which the hydrostatic force is applied, and are labeled as " $A$." The cut out planar areas have pieces of foam in the form 
of the pressure distribution attached to them. Arrows are drawn on the sides of the foam to represent the pressure distribution. The trapezoid on the side of the purple piece of foam representing the pressure distribution acting on the rectangular plane has recently had an " $A_{T}$ " label added to it. Both cut out shapes are placed in the model before the demonstration begins, and 'hidden' from view behind the blue triangle.

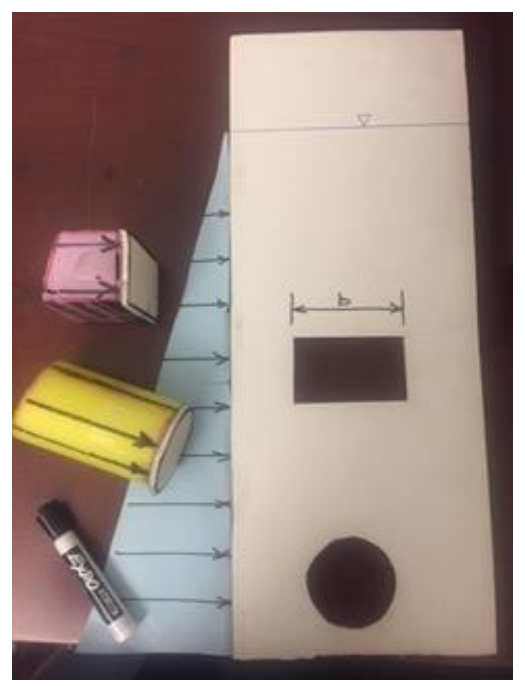

Figure 4. Pieces of physical model used in class

At the beginning of the demonstration, students are shown the white face of the model with both planar areas $(A)$ and the blue triangle. The blue triangle is then pivoted out of the way to reveal the foam models of the pressure distribution that are behind the rectangular and circular cut-outs. Discussion of the differences between " $A$ " and " $A_{T}$ " ensues. Although the arrows on the sides of both foam blocks initially appear to represent trapezoids, especially at a distance, once the cutouts with attached foam are removed for inspection, students more clearly see that only the purple block has a planar side that can be labeled " $A_{T}$." The absence of a similar " $A_{T}$ " on the curved side of the yellow foam attached to the circular plane demonstrates that " $A_{T}$ " is only defined if the plane on which the pressure is acting has constant width. This reinforces the lesson that equation (1) is not always appropriate to use. As explained below, in recent semesters this has also led to discussion about the correct definition of a prism, to support the discussion of when it is appropriate to calculate the volume of a pressure prism.

With limitations, the model can also be used to explain how pressure is calculated if the plane is inclined. The author demonstrates how $A$ remains unchanged if the plane is inclined; however, since the size of the blue triangular pressure distribution is fixed, the demonstration can lead to misunderstanding. The author therefore currently resorts to the board to explain this concept and hopes to build another model which can be used for this purpose.

Assessment of benefits of the physical model

Students have generally provided immediate positive feedback about the physical model during and after the class when it is used. There is often a sense of surprise when I reveal it and begin 
the demonstration. In addition to providing a means of clarifying the calculations at hand, the use of the model also tends to engage the students more.

A formal assessment of the impact of the model on student grades was not done. This would have required more granular breakdown of student performance on subsections of assigned problems, since computation of the resultant force is usually just a component of the grade for a problem. This problem subsection breakdown was not recorded before the model was introduced, so even recording problem subsection grades after the model was introduced would not have sufficed.

Furthermore, electronic records of student grades on a problem-by-problem basis for several semesters before the model was built were lost, so there is little data to compare before and after grades for specific problems that are similar to each other. Overall grades on entire problem sets were compared instead. The problem sets typically included additional types of hydrostatics problems, not just problems involving resultants on flat plates. Student averages on hydrostatics problem sets during three semesters before the model was introduced ranged from 60 to $63 \%$; after the model was introduced, student averages on similar problem sets ranged from $59 \%$ to $71 \%$ over five semesters, with an average of $67 \%$. The grade of $59 \%$ is an outlier; eliminating it yields a range of $67 \%$ to $71 \%$ on four problem sets, with an average of $69 \%$. This analysis is evidence that student performance on hydrostatics problems sets generally improved after the model was introduced. However, it should not be used as strong evidence that the physical model was the reason. (Perhaps it is evidence that the ExCEEd workshop improved overall teaching.)

A simple survey was emailed to 55 former students from three recent semesters to assess the usefulness of the model. The questions focused on whether the model improved understanding of the calculations. Responses are shown in Figure 5.

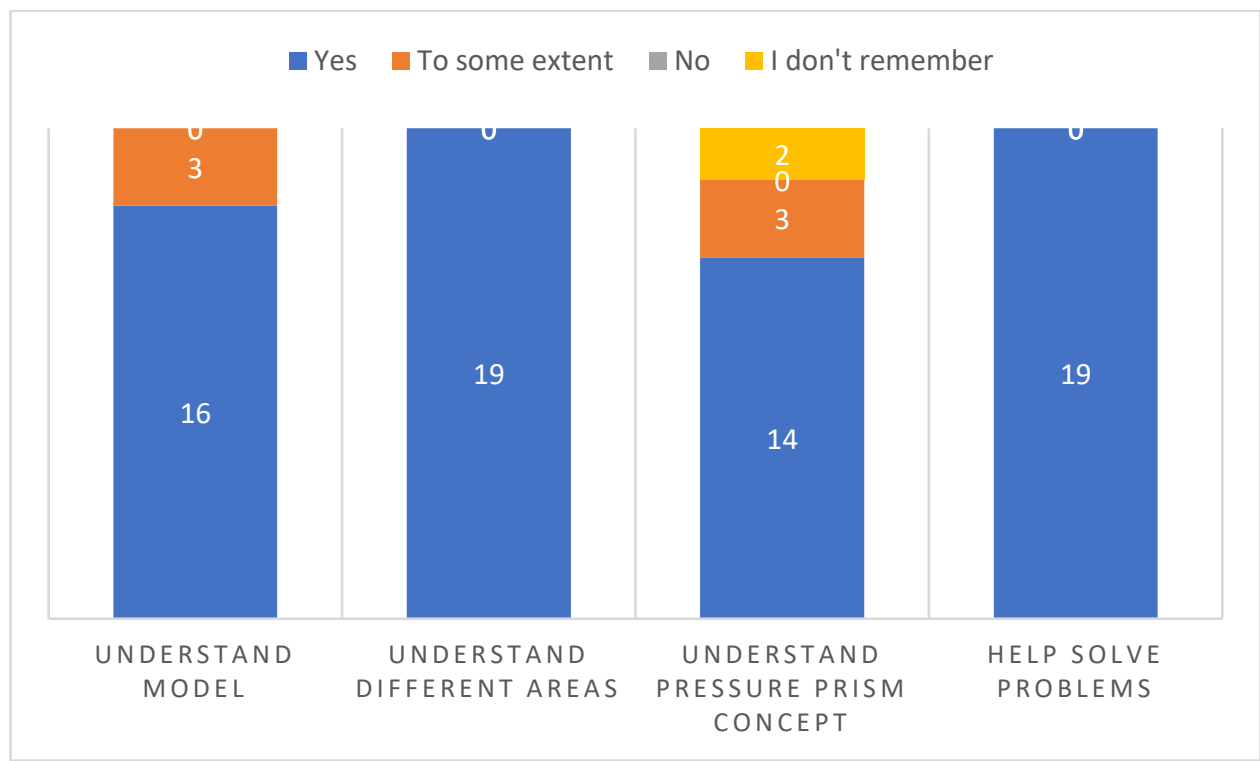

Figure 5: Student responses to survey about physical model effectiveness 
Of the 19 students who responded, three admitted that they didn't quite understand the model when it was presented. Nevertheless, there was unanimous agreement that the model helped students understand the differences between the two areas denoted as $A$ and $A_{T}$ in this paper and that the model also helped them solve problems. Only 14 students responded that they understood the pressure prism concept. I believe part of the problem is that students are unclear about the exact definition of a prism; some appear to believe that any three-dimensional solid is a prism so they do not understand why the volume behind the circle is not considered a prism. During recent semesters I have made an effort to clearly define a prism; however, the sample size is too small to determine if students in recent semesters have a better understanding.

Students were also asked to provide open-ended feedback. The six students who opted to do so were unanimous in stating the model's benefits. Three sample responses are presented below:

"I am definitely more of a visual / hands on learner and I know a topic like this is not the easiest thing to get across, ... so I think that by having this physical 3 dimensional visual it definitely helped further my understanding."

"At the time of the demonstration, it was unclear. After applying the knowledge to practice questions, it was very helpful seeing it. Especially, for it being tangible."

"Sometimes you can't draw concepts on a board - Prof Ronan's 3D model was a great visual in learning pressure distributions in 3D."

\section{Conclusions}

A simple model representing the hydrostatic pressure distribution acting on a planar surface is used to assist teaching students how to calculate the resultant force. The model has received positive feedback from students; it helps students understand the terms used in relevant equations which improves their ability to solve problems. The use of the model during class also engages students more during the lesson. 